\title{
BMJ Open Cost of oropharyngeal dysphagia after stroke: protocol for a systematic review
}

\author{
Sergio Marin, ${ }^{1}$ Mateu Serra-Prat, ${ }^{2,3}$ Omar Ortega, ${ }^{3,4}$ Pere Clavé ${ }^{3,4}$
}

To cite: Marin S, SerraPrat M, Ortega 0 , et al. Cost of oropharyngeal dysphagia after stroke: protocol for a systematic review. BMJ Open 2018;8:e022775. doi:10.1136/ bmjopen-2018-022775

- Prepublication history for this paper is available online. To view these files, please visit the journal online (http://dx.doi. org/10.1136/bmjopen-2018022775).

Received 20 March 2018 Revised 18 0ctober 2018 Accepted 26 October 2018

A) Check for updates

C Author(s) (or their employer(s)) 2018. Re-use permitted under CC BY-NC. No commercial re-use. See rights and permissions. Published by BMJ.

${ }^{1}$ Pharmacy Department, Consorci Sanitari del Maresme. Hospital de Mataró., Mataro,

Spain

${ }^{2}$ Research Unit, Consorci Sanitari del Maresme, Barcelona, Spain

${ }^{3}$ Centro de Investigación Biomédica en Red de enfermedades hepáticas y digestivas (CIBERehd), Instituto de Salud Carlos III, Barcelona, Spain

${ }^{4}$ Gastrointestinal Physiology Laboratory, Consorci Sanitari del Maresme, Universitat Autónoma de Barcelona, Catalunya, Spain

Correspondence to

Sergio Marin; smarin@csdm.cat

\begin{abstract}
Introduction Oropharyngeal dysphagia (OD) is a major disorder following stroke. OD can produce alterations in both the efficacy and safety of deglutition and may result in malnutrition, dehydration, frailty, respiratory infections and pneumonia. These complications can be avoided by early detection and treatment of OD in poststroke patients, and hospital stays, medication and mortality rates can be reduced. In addition to acute in-hospital costs from OD complications, there are other costs related to poststroke OD such as direct non-healthcare costs or indirect costs.

The objective of this systematic review is to assess and summarise literature on the costs related to $\mathrm{OD}$ in poststroke patients.
\end{abstract}

Methods and analysis A systematic review of studies on the cost of OD and its complications (aspiration, malnutrition, dehydration, aspiration pneumonia and death) in patients who had a stroke will be performed from the perspectives of the hospital, the healthcare system and/ or the society. The main outcomes of interest are the costs related to poststroke OD. We will search MEDLINE, Embase and the National Health Service Economic Evaluation Database. Studies will be included if they are partial economic evaluation studies, studies that provide information on costs in adult ( $>17$ years) poststroke patients with $\mathrm{OD}$ and/or its complications (malnutrition, dehydration, frailty, respiratory infections and pneumonia) or economic evaluation studies in which the cost of this condition has been estimated. Studies will be excluded if they refer to oesophageal dysphagia or OD caused by causes other than stroke. Main study information will be presented and summarised in tables, separately for studies that provide incremental costs attributable to $\mathrm{OD}$ or its complications and studies that report the effect of $\mathrm{OD}$ or its complications on total costs of stroke, and according to the perspective from which costs were measured.

Ethics and dissemination The results of this systematic review will be published in a peer-reviewed journal. PROSPERO registration number CRD42018099977.

\section{INTRODUCTION}

Oropharyngeal dysphagia (OD) is a major disorder following stroke with a high incidence in acute poststroke patients $(37 \%-$ $78 \%) .{ }^{1}$ OD can improve after the first weeks of the stroke, however it persists in as many as $50 \%$ of patients. ${ }^{2}$ OD, which is classified in the International Classification of Diseases 10th Revision promoted by the WHO, ${ }^{3}$ can produce alterations in both the efficacy and
Strengths and limitations of this study

- This systematic review protocol was performed using the Preferred Reporting Items for Systematic Reviews and Meta-Analyses recommendations.

- The proposed methodology will allow us to assess the quality of the selected studies.

- The bibliographical search considers MEDLINE and Embase databases.

- Unpublished material and abstracts will not be included in this systematic review.

- Costs are very context-specific so they are difficult to summarise in a single result.

the safety of deglutition. Impaired efficacy of swallow causes malnutrition and/or dehydration in up to $25 \%$ of patients and impaired safety of swallow may lead to aspiration pneumonia with high mortality rates. ${ }^{145}$ However, still today, most patients do not receive comprehensive care, and poststroke OD is an underdiagnosed and undertreated condition worldwide. OD is an inexpensive complication to diagnose. For OD diagnosis, well-established methods and tools exist, such as routine screening followed by expert assessment. Position Statements of the European Society for Swallowing Disorders recommend that all patients who had a stroke should be screened for OD with available, easy to use and validated screening tools. ${ }^{6}$ Evidence-based and effective treatment for OD is mainly oriented to compensating swallow impairments through adaptation of fluid viscosity and solid food textures to avoid aspiration and choking, and improving nutritional status and oral health to avoid respiratory infections. Advances in treatment are mainly focused on peripheral stimulation strategies and central, non-invasive stimulation strategies. Among these methods, transcutaneous and intrapharyngeal electrical stimulation, pharmacological stimulation through transient receptor potential channels of the vanilloid subtype agonists and non-invasive brain stimulation techniques, repetitive transcranial magnetic stimulation and transcranial direct current stimulation 
are emerging treatments for swallow dysfunction among poststroke patients. ${ }^{7}$ The aim of these interventions is to restore the swallow function.

Screening and treatment of malnutrition and dehydration are also two important challenges for these patients in order to avoid complications related with malnutrition such as pressure sores. ${ }^{8}$ Moreover, screening and promotion of good oral health practices among these patients should be implemented to reduce bacterial colonisation by respiratory pathogens..$^{9} 10$

Regardless of its aetiology, OD has been related to longer length of stay, and higher inpatient costs, likelihood of being transferred to postacute care facility and inpatient mortality during hospitalisation. ${ }^{11}$ Early detection and treatment of OD in poststroke patients can diminish these complications and reduce hospital stays, readmissions, aspiration pneumonia and mortality rates, as well as the use of some medication such as antibiotics. ${ }^{12}$ Taking into account all these secondary complications related to poststroke $\mathrm{OD}$, this condition could have a high economic impact on healthcare costs. The correct treatment of this condition could lead to considerable savings in healthcare costs. In addition to acute in-hospital costs due to OD complications, there are other costs related to poststroke OD such as rehabilitation care, institutionalisation, direct non-healthcare costs and indirect costs due to loss of productivity. The chronic nature of OD means medical costs related to patient care outside the acute hospital setting must be taken into account. Knowing costs related to this poststroke disorder can lead to a better understanding of its impact. Our hypothesis is that the impact of OD on the health-economic and social costs of stroke is high and that minimal care of OD is economically sounder than low care of OD. Therefore, our aim is to quantify the cost of acute and chronic poststroke OD. It is important to understand the economic burden in order to change clinical practice. However, costs associated to acute and chronic OD in patients who had a stroke have been poorly studied and are not well known. One study has shown that presenting OD after stroke was associated with high mortality rates during hospital stay and was an independent risk factor for prolonged length of hospital stay and to be institutionalised after hospital discharge; OD was also an independent risk factor for poorer functional capacity and increased risk of mortality 3 months after the stroke episode. This study stated these factors were of great importance not only from the perspective of patient health, but also because of their social and economic burden. ${ }^{13}$ The objective of this systematic review is to assess and summarise all the knowledge on the costs related to OD in poststroke patients.

\section{METHODS AND ANALYSIS}

A systematic review of studies on the cost of OD and its complications (aspiration, malnutrition, dehydration, aspiration pneumonia or death) in patients who had a stroke will be performed from hospital, healthcare system and social perspectives. The main outcome of interest is the costs related to poststroke OD. This systematic review will be performed during 2018.

\section{Preferred Reporting Items for Systematic Reviews and Meta- Analyses statement}

Systematic reviews are fundamental instruments to assess all the evidence related to a topic precisely and trustworthily. Nowadays, systematic reviews are key tools in updating the knowledge on a certain topic, achieving conclusions on available evidence and taking decisions in the healthcare environment. Because of this, systematic reviews must follow an exhaustive and accurate methodology and need to be reported with clarity and transparency. For this reason, we will use the methodology proposed by Preferred Reporting Items for Systematic Reviews and Meta-Analyses (PRISMA) to carry out this systematic review. ${ }^{14}$ In this protocol, PRISMA Protocols annex (PRISMA-P) has been used. PRISMA-P is a specific PRISMA section to develop protocols. It can be used as a guideline to develop protocols for systematic reviews according to PRISMA methodology. Moreover, PRISMA-P offers study examples for each item included in the protocol. These examples are extracted from studies that have been relevant in their respective fields, reported with high quality and carried out using accurate methodology. ${ }^{15}$ We need to use protocols to increase work quality and reduce to the maximum the risk of bias secondary to mistaken internal methodology and inaccurate reporting. Protocols are key tools for developing systematic reviews that can lead to reliable results to help in decision-making, improve backup for future investigation and serve as a summary of the available evidence on a certain topic. For all these reasons, we use PRISMA as a reference in this work.

\section{Literature search}

We will search MEDLINE using PubMed and Embase using Ovid. We will search on the National Health Service Economic Evaluation Database as well using the Center for Reviews and Dissemination Database of the University of York. The MeSH and search terms used in the search strategy and their combination are described in table 1 . Using this search strategy in MEDLINE using PubMed, a total of 70 articles were found in June 2018. No publication date and no language restrictions will be imposed. Unpublished material and abstracts will not be included in this systematic review.

\section{Selection process}

We will analyse all the studies identified through the literature search described in this protocol using a doublephase process: an initial screening phase and a subsequent selection phase where studies will be included according to the review eligibility criteria. The references of the studies included will be checked for additional eligible studies.

In the initial screening phase, the abstract and title of the studies will be analysed to eliminate studies not containing data on costs in poststroke OD, its 
Table 1 Search terms and MeSH terms used in the bibliographical search

\begin{tabular}{|c|c|c|}
\hline $\begin{array}{l}\text { Terms related to oropharyngeal } \\
\text { dysphagia and connected among } \\
\text { themselves by 'OR' }\end{array}$ & $\begin{array}{l}\text { Terms related to stroke and connected } \\
\text { among themselves by 'OR' }\end{array}$ & $\begin{array}{l}\text { Terms related to health economics and } \\
\text { connected among themselves by 'OR' }\end{array}$ \\
\hline 1. Oropharyngeal Dysphagia.tw. & 21. "Stroke'[Mesh] & 28. "Economics'[Mesh] \\
\hline 3. Dysphagia therapy/ & 23. Post-stroke/ & 30. 'Models, Economic'[Mesh] \\
\hline 4. 'Deglutition'[Mesh] & 24. 'Stroke Rehabilitation’ [Mesh] & 31. Cost effective* \\
\hline 5. Deglutition Disorders'[Mesh] & 25. 'Brain Ischemia/complications'[Mesh] & 32. Cost[WORD] \\
\hline 7. 'Oropharynx/diagnosis'[Mesh] & 27. 'Cerebral Hemorrhage'[Mesh] & 34. 'Health Resources'[Mesh] \\
\hline $\begin{array}{l}\text { 8. 'Oropharynx/diagnostic } \\
\text { imaging'Mesh] }\end{array}$ & & $\begin{array}{l}\text { 35. 'Tertiary Care Centers/ } \\
\text { economics'[Mesh] }\end{array}$ \\
\hline 9. 'Oropharynx/pathology'[Mesh] & & 36. 'Hospitalization/economics'[Mesh] \\
\hline 10. 'Oropharynx/pharmacology'[Mesh] & & 37. .'Rehabilitation Centers/ \\
\hline 11. 'Oropharynx/physiopathology'[Mesh] & & economics'[Mesh] \\
\hline $\begin{array}{l}\text { 16. 'Pneumonia/prevention and } \\
\text { control'[Mesh] }\end{array}$ & & $\begin{array}{l}\text { 41. 'Emergency Medical Services/ } \\
\text { economics' [Mesh] }\end{array}$ \\
\hline 17. 'Nutritional Status'[Mesh] & & 42. 'Food, Formulated/economics'[Mesh] \\
\hline 18. 'Nutrition Assessment'[Mesh] & & 43. 'Cerebrovascular Disorders/ \\
\hline 19. 'Malnutrition'[Mesh] & & economics'[Mesh] \\
\hline 20. Enteral Nutrition* & & \\
\hline
\end{tabular}

Terms detailed in the three columns above, related to oropharyngeal dysphagia, stroke and health economics, will be connected using 'AND'.

complications (malnutrition, dehydration, frailty, respiratory infections and pneumonia) or relevant data or information. In the abstract or title, there must appear an economic term such as 'costs' or 'resources consumption' and 'dysphagia' or 'malnutrition, dehydration, frailty, respiratory infections and pneumonia' with 'stroke'. This selection process will be done by one sole reviewer and, subsequently, a second reviewer will check the eliminated abstracts.

In the second selection phase, studies will be included in the systematic review if they have partial economic evaluation, studies that provide information about costs in adult (>17years) poststroke patients with OD and/ or its complications (malnutrition, dehydration, frailty, respiratory infections and pneumonia) or economic evaluation studies in which the cost of OD has been estimated. Studies will be excluded if they are (a) oesophageal dysphagia studies, (b) studies not related to OD, (c) studies in which OD is related to a cause other than stroke, (d) duplicate publications of the same study or (e) other causes (explained above). This information will be presented in a content table. Full text of selected studies will be carefully assessed according to a pre-established data collection notebook. Figure 1 summarises the selection process. Two independent reviewers will participate in this selection process. In case of disagreement over one or more studies, a third reviewer will review the study and a final consensus will be made. The reason for excluding the study will be recorded. No restrictions related to the size of the sample will be imposed.

\section{Data collection}

Two reviewers will extract data from the selected studies and will register it in a standard data collection form. In case of disagreement between them, a third reviewer opinion will be required to reach an agreement and to take a final consensus decision. If necessary, we will contact authors of included studies in order to ask for unreported information or to clarify possible misunderstandings. Data directly obtained from the authors will be clearly identified. We will report any assumption resulting from lost or unavailable information. To manage study data, we will transfer all information in the data collection form to a spreadsheet. Data gathering will refer to main study characteristics, quality assessment and study results. The information to be obtained from each study is presented below:

a. Study identification: first author, journal of publication and year of publication.

b. Main design characteristics: type of study (cost of illness study or another type of study that provides cost of illness information in this field), epidemiological approach (cross-sectional or longitudinal), retrospective or prospective data gathering, perspective of the analysis (hospital, patient, healthcare system, societal or insurance carrier perspective); time horizon, use of temporary discount rate, sensitivity analysis (yes/no), presence of a control group (patients not affected by OD), location/setting.

c. Study sample characteristics: sample size; sociodemographic data (age, average and range; gender), patient 


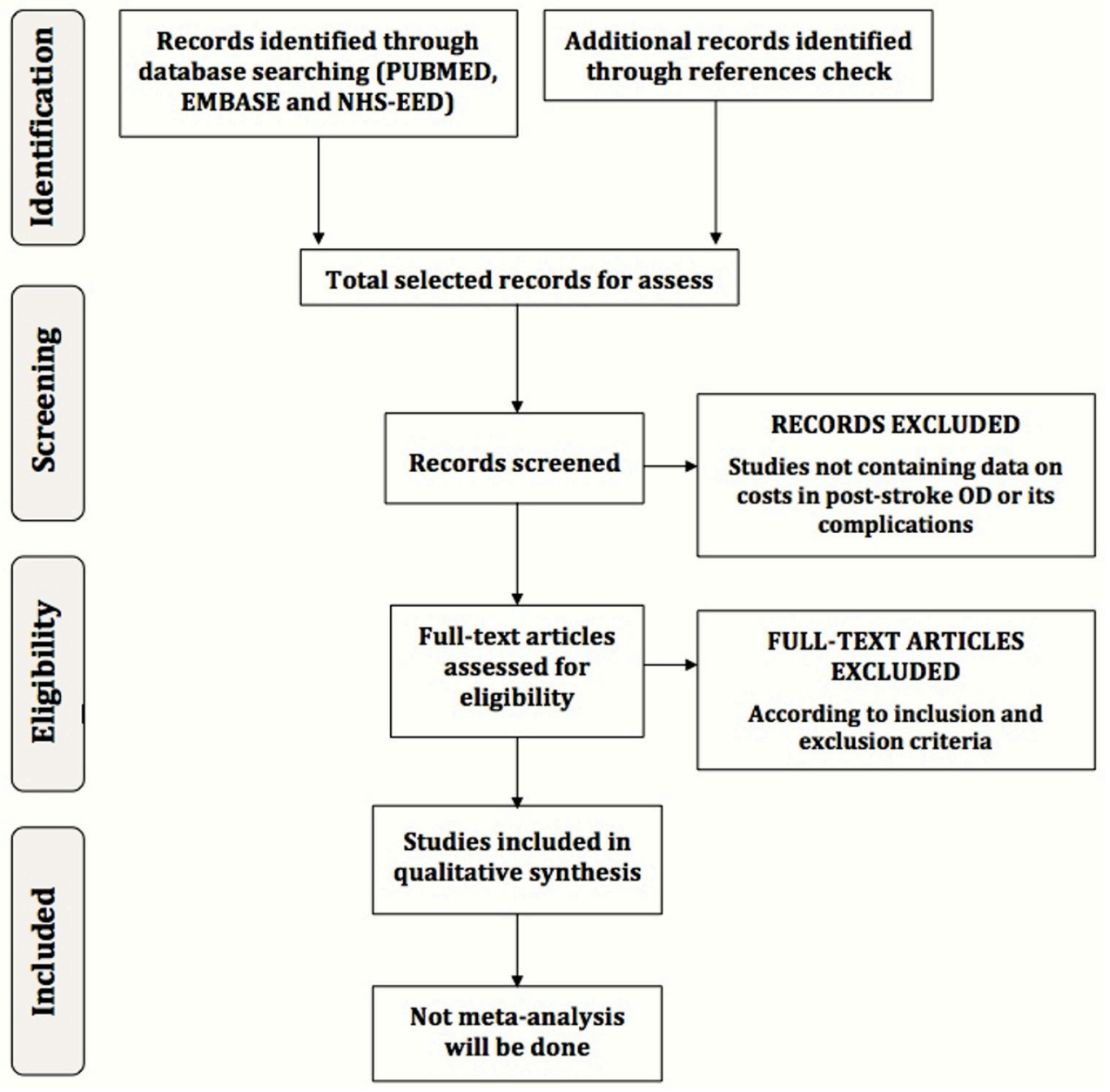

Figure 1 Selection process flow diagram. NHS-EED, National Health Service Economic Evaluation Database; OD, oropharyngeal dysphagia.

inclusion and exclusion criteria, patient's functional capacity (Rankin, Barthel), patient's comorbidities (Charlson), stroke type (ischaemic or haemorrhagic), National Institutes of Health Stroke Scale (NIHSS) and/or Canadian Neurologic Scale on admission and on discharge, fibrinolysis treatment (yes/no), endovascular treatment (yes/no), method used to diagnose OD (videofluoroscopy, fibre optic endoscopic evaluation of swallowing, volume-viscosity swallowing test or other bedside methods), nutritional assessment, discharge destination (rehabilitation ward, nursing home, domicile).

d. Elements of cost considered: direct healthcare costs (hospital ward, intensive care unit, emergency room, institutionalisation in a nursing home, primary care visits and to nutritionists, physical therapists, speech therapists, ambulance, medication, diagnostic tests, special diets, tube-feeding, percutaneous endoscopic gastrostomy (PEG) insertion, antibiotic consumption, pneumonia-related costs, etc), direct non-health care costs (social services and transportation costs) and indirect costs (loss of productivity).

e. Data source: medical registries, MEDICARE databases, national patient databases, insurance databases, data collected from individual research groups, data collected from individual hospitals.

f. Study results: the primary outcome will be the costs related to OD in poststroke patients reported in monetary units $(€)$. If the studies provide specific breakdown of costs, we will report this information (direct hospital costs, rehabilitation care costs, direct non-healthcare costs, indirect costs and productivity loss, and intangible costs). We will also collect data on quantities of health and social resource consumption, currencies used and whether the study shows total or incremental costs. Whenever possible, the cost adjusted for the severity of the stroke (eg, based on NIHSS or Canadian scale) or other confounding factors will be considered.

Quality assessment, risk of bias in individual studies, meta bias and confidence in cumulative evidence

Methodological quality and risk of bias and meta bias will be evaluated using Drummond's checklist for assessing economic evaluations. ${ }^{16}$ This checklist provides a set of items applicable to a critical appraisal of health economic evaluation studies and includes the following three domains: study design, data collection and analysis and interpretation of results. Each checklist domain has 
different sections; there are 10 sections containing questions on the study, and every section will be rated as 'yes/ partly/no'. A fourth option as 'not available/not applicable' has been added since not all sections are adapted to studies like cost of illness studies. Drummond's original list consists of 35 points but we have adapted it and will only use the 25 points applicable to cost studies. We will give a global score for the quality of each study which we calculate dividing the total number of points rated as 'yes' between the total points applicable for each study, and record it as a percentage.

We will assess confidence and strength of evidence in this systematic review using the Grading of Recommendations Assessment, Development and Evaluation (GRADE) methodology. ${ }^{17}$ GRADE is a tool designed to assess the strength of the summarised evidence across studies in systematic reviews by evaluating both study limitations, imprecision, inconsistency of results, indirectness of evidence, publication bias, magnitude of the effect and the presence of confounders that minimise the effect. Finally, we will rate the strength of evidence across studies as high, moderate, low or very low, and we will make an evidence profile with a detailed quality assessment.

\section{Data synthesis}

A systematic meta-narrative synthesis will be made, so, we will present the results in narrative form. Findings and characteristics of the included studies will be summarised and explained in text and tables. We will present results in the text following this order: (1) data on costs related to poststroke OD, (2) data on costs related to OD complications in this order: (a) aspiration, (b) malnutrition, (c) dehydration, (d) aspiration pneumonia and (e) death. Data will be presented separately for those studies that provide incremental costs attributable to OD or its complications from those studies that report the effect of OD or its complications on total costs of stroke, and according to the perspective in which costs are measured (hospital, patient, healthcare system, societal or insurance carrier). Main study information will be presented and summarised in several tables of evidence. This presentation will be performed separately in the same way as in tables. No conversion of study data will be performed, and all data will be reported in the original format during the initial presentation of the results. One set of tables of evidence will present main designs and sample characteristics as well as cost elements considered and data source. Another set of tables will report data on main results and quality assessment.

A weighted mean cost of poststroke OD will be estimated for those studies with the same perspective and time horizon and with similar design characteristics. Mean cost will be weighted by sample size. No other quantitative methods of synthesising data will be performed. Moreover, evidence obtained from studies will be synthesised through a qualitative synthesis method, using a meta-narrative method. In this section, we will take into account the risk of bias information obtained from each study. No study will be eliminated based on its risk of bias, but we will assess how risk of bias can affect the main results and outcome measures. To present this data synthesis of results correctly, we will follow the recommendations stated in the guidance from the Centre for Reviews and Dissemination. ${ }^{18}$

\section{Glossary of terms in health economics}

- Economic evaluation in healthcare: analytical methodology that aims to compare the costs and the consequences in health of various alternatives (interventions, treatments, programmes, etc).

- Partial economic evaluation: economic evaluation technique that only compares the costs of the various alternatives studied but does not consider their effects on health. Also called cost analysis.

- Analysis perspective: point of view from which the analysis is focused and which determines the cost elements to consider. Each perspective provides specific information for a particular entity, for example, hospital perspective mainly includes acute in-hospital costs while healthcare system perspective includes costs related to those medical costs beyond the acute in-hospital stay. The societal perspective is the most complete because it includes healthcare and non-healthcare costs and loss of productivity.

- Type of costs: cost studies classify costs as direct costs, indirect costs and intangible costs.

- Direct medical costs are those related to a healthcare intervention (eg, hospital ward or medication) and direct non-medical costs are those associated with provision of medical services (eg, transportation costs).

- Indirect costs are those related to productivity loss, morbidity, mortality or time spent.

- Intangible costs are rarely studied and are those related to suffering and pain related to a disease or treatment.

- Discount rate: there is a preference to obtain benefits straight away and to delay costs. When the time horizon is longer than 1year, a temporary discount rate should be used to allow costs and results that will occur over time to be measured at present values. An annual $3 \%-5 \%$ discount rate is usually used.

- Sensitivity analysis: some decisions in the economic analysis are based on uncertain data. The sensitivity analysis is aimed attesting the robustness of results of the economic evaluation when changing the assumed values of some variables used in the analysis. ${ }^{149}$

\section{PATIENT AND PUBLIC INVOLVEMENT STATEMENT}

There was no public or patient involvement in the elaboration of this protocol.

\section{ETHICS AND DISSEMINATION}

The results of this systematic review will be published in a peer-reviewed journal. This systematic review is the first part of a research project aiming to evaluate the health economic and social costs of OD in patients with had a stroke to better 
understand and raise awareness on minimal care for this common and severe complication. Complications of OD are related to three main aspects: (a) impaired safety of swallow, causing the aspiration of respiratory pathogens to the airway; (b) impaired nutritional status, leading to malnutrition, impaired immunity and frailty and (c) poor oral health and hygiene, associated with oral colonisation by respiratory pathogens. It is necessary to treat these three aspects simultaneously with the aim of maximising the number of patients treated with simple and cost-effective measures based on the best scientific evidence. This minimal and massive intervention is based on fluid and food texture adaptations, nutritional supplementation and oral hygiene. ${ }^{20}$ The full extent of this project will include (a) a systematic review of the literature on the cost of OD after stroke, (b) a systematic review of the literature on full economic evaluations of interventions related to OD, (c) a health economic analysis of a study on the prevalence and evolution of OD in patients with stroke (with 1 year follow-up) to assess the cost of OD and (d) a study on the cost-effectiveness of compensatory versus active interventions (those treatments for OD that aim to restore the impaired swallow function) to improve swallowing function in these patients. OD treatment is moving from compensatory strategies towards promoting brain plasticity, both to recover swallow function and to improve brain-related swallowing dysfunction. ${ }^{7}$

Acknowledgements We would like to thank Agustí Viladot, member of Mataró Hospital library service, for helping with search strategy and Jane Lewis for reviewing the English. This work has been conducted within the framework of a doctoral thesis in medicine from the Medicine Department of the Autonomous University of Barcelona.

Contributors SMR is the guarantor. He drafted the first version of this manuscript. He provided expertise on health economics. He contributed to the development of the introduction (rationales and objectives), the eligibility criteria, the selection of the information sources, the search strategy, the selection process, the quality assessment, the data extraction criteria and the data synthesis. He read and approval the final revision of this protocol. MS-P provided expertise on investigation methodology and health economics. He provided important references for the development of the methodology of this work. He reviewed and made contributions on the methodology of all the sections of this protocol. He reviewed the correct use of all the economic terms included in this manuscript. He collaborated in writing the manuscript. $00 \mathrm{~F}$ provided expertise on poststroke oropharyngeal dysphagia (OD) treatments and interventions. He contributed on the development of the data collection, quality assessment and data synthesis sections. He provided a critical revision of all the sections of this protocol. He collaborated in writing the manuscript. He collaborated in writing the manuscript. PCC provided expertise on poststroke OD. He reviewed the correct use of all the medical terms included in this manuscript. He contributed on the development and writing of the introduction. He provided a critical revision of all the sections of this protocol. He contributed to the correct following of the recommendations proposed by Preferred Reporting Items for Systematic Reviews and Meta-Analyses. All authors provided a critical revision and read and approval the final revision of this protocol.

Funding This study is funded by an educational grant from Nutricia Advanced Medical Nutrition. This study is supported by Fundació Salut del Consorci Sanitari del Maresme, Ciberehd (in turn supported by the Instituto de Salud Carlos III, Barcelona, Spain) and Fundació de Recerca en Gastroenterologia (FUREGA).

Disclaimer The sponsor has no role in the development of this study.

Competing interests None declared.
Patient consent for publication Not required.

Provenance and peer review Not commissioned; externally peer reviewed.

Open access This is an open access article distributed in accordance with the Creative Commons Attribution Non Commercial (CC BY-NC 4.0) license, which permits others to distribute, remix, adapt, build upon this work non-commercially, and license their derivative works on different terms, provided the original work is properly cited, appropriate credit is given, any changes made indicated, and the use is non-commercial. See: http://creativecommons.org/licenses/by-nc/4.0/.

\section{REFERENCES}

1. Martino R, Foley N, Bhogal S, et al. Dysphagia after stroke: incidence, diagnosis, and pulmonary complications. Stroke 2005;36:2756-63.

2. Mann G, Hankey GJ, Cameron D. Swallowing function after stroke: prognosis and prognostic factors at 6 months. Stroke 1999;30:744-8.

3. WHO. International classification of disease [online]. http://www.who. int/ classifications/icd/en (accessed Feb 2018).

4. Clavé P, de Kraa M, Arreola V, et al. The effect of bolus viscosity on swallowing function in neurogenic dysphagia. Aliment Pharmacol Ther 2006;24:1385-94.

5. Cook IJ, Kahrilas PJ. AGA technical review on management of oropharyngeal dysphagia. Gastroenterology 1999;116:455-78.

6. Schepp SK, Tirschwell DL, Miller RM, et al. Swallowing screens after acute stroke: a systematic review. Stroke 2012;43:869-71.

7. Cabib $\mathrm{C}$, Ortega $\mathrm{O}$, Kumru $\mathrm{H}$, et al. Neurorehabilitation strategies for poststroke oropharyngeal dysphagia: from compensation to the recovery of swallowing function. Ann N Y Acad Sci 2016;1380:121-38.

8. Geeganage C, Beavan J, Ellender S, et al. Interventions for dysphagia and nutritional support in acute and subacute stroke. Cochrane Database Syst Rev 2012;10:CD000323.

9. van der Maarel-Wierink CD, Vanobbergen JN, Bronkhorst EM, et al. Oral health care and aspiration pneumonia in frail older people: a systematic literature review. Gerodontology 2013;30:3-9.

10. Seedat J, Penn C. Implementing oral care to reduce aspiration pneumonia amongst patients with dysphagia in a South African setting. S Afr J Commun Disord 2016;63.

11. Patel DA, Krishnaswami S, Steger E, et al. Economic and survival burden of dysphagia among inpatients in the United States. Dis Esophagus 2018;31:1-7.

12. Ickenstein GW, Riecker A, Höhlig C, et al. Pneumonia and in-hospital mortality in the context of neurogenic oropharyngeal dysphagia (NOD) in stroke and a new NOD step-wise concept. J Neurol 2010;257:1492-9.

13. Rofes L, Muriana D, Palomeras E, et al. Prevalence, risk factors and complications of oropharyngeal dysphagia in stroke patients: a cohort study. Neurogastroenterology \& Motility 2018;30:e13338.

14. Liberati A, Altman DG, Tetzlaff J, et al. The PRISMA statement for reporting systematic reviews and meta-analyses of studies that evaluate healthcare interventions: explanation and elaboration. BMJ 2009;339:b2700.

15. Shamseer L, Moher D, Clarke M, et al. Preferred reporting items for systematic review and meta-analysis protocols (PRISMA-P) 2015: elaboration and explanation. BMJ 2015;350:g7647.

16. Drummond MF, O'Brien B, Torrance GW, et al. Methods for the economic evaluation of health care programmes. 2 nd ed. Oxford: Oxford University Press, 1997.

17. Guyatt G, Oxman AD, Akl EA, et al. GRADE guidelines: 1. Introduction-GRADE evidence profiles and summary of findings tables. J Clin Epidemiol 2011;64:383-94.

18. Centre for Reviews and Dissemination. Systematic reviews: CRD's guidance for undertaking reviews in health care. Centre for Reviews and Dissemination, University of York: York, 2009.

19. Jo C. Cost-of-illness studies: concepts, scopes, and methods. Clin Mol Hepatol 2014;20:327-37.

20. Martín A, Ortega O, Roca M, et al. Effect of A Minimal-Massive Intervention in Hospitalized Older Patients with Oropharyngeal Dysphagia: A Proof of Concept Study. J Nutr Health Aging 2018;22:739-47. 